\title{
Welcome Editorial: Change and Continuity in Phonetica
}

\author{
Catherine T. Best \\ MARCS Institute, and the School of Humanities and Communication Arts, \\ University of Western Sydney, Penrith, Australia
}

This first issue of volume 71 of Phonetica marks the official start of my term as Editor. I take on this new challenge with enthusiasm and humility, on the understanding that publication of quality papers on current interdisciplinary research and theory in the phonetic sciences is essential to progress in the field and fosters scientific discussion among its members.

First, I'd like to acknowledge the many people who have supported and continue to support this new phase for Phonetica. I am privileged to share stewardship of this esteemed journal with an outstanding scientific team (http://www.karger.com/Journal/ EditorialBoard/224275). The 2014 slate of Associate Editors and Board members represents leading-edge phonetics research from all corners of the world and across the array of disciplines and interdisciplinary approaches reflected in Phonetica's communicative phonetic science perspective (see next paragraph). The team provides a balance of new and continuing members, thus offering a combination of fresh perspective and extensive editorial experience and knowledge of Phonetica. I am excited to be working with them - I am pleased that each has agreed to join me in taking Phonetica forward, and look forward to collaborating with them on the journey. I am also grateful to the Phonetica staff at Karger Publishers, who manage the processing of submitted manuscripts, production of published issues, promotion, and key aspects of communication with the scientific team (http://www.karger.com/Journal/Contact/224275). They are excellent, dedicated, and have been enormously helpful. Their support was crucial especially during the editorial transition period that began in September 2013, which also marked a shift to a new online system for manuscript submission and processing for Phonetica. Last and yet foremost, I thank Klaus Kohler, the outgoing Editor, for inviting me to assume the editorship of Phonetica. It is both an honour and a pleasure to take up the reins on this wonderful interdisciplinary forum for the communicative phonetic sciences that Professor Kohler and his editorial teams have created. I particularly appreciate his insightful and informative background on the journal's history, mission and operations, as well as his help in making the editorial transition smooth. It am grateful as well that he served as Editor for two of the papers appearing in this first issue of 2014 [Pavlík, 2014; Tyler et al., 2014], and for his quick and supportive answers to the questions I have posed to him while learning to be Editor of Phonetica. I wish him a wonderful and well-deserved retirement, enjoying all the things he has been looking forward to doing.

\begin{tabular}{|c|c|}
\hline KARGER & $\begin{array}{l}\text { (C) } 2014 \text { S. Karger AG, Basel } \\
0031-8388 / 14 / 0711-0001 \\
\$ 39.50 / 0\end{array}$ \\
\hline $\begin{array}{l}\text { ger.co } \\
\text { lo }\end{array}$ & \\
\hline
\end{tabular}

Catherine T. Best

MARCS Institute, and the School of Humanities and Communication Arts, University of Western Sydney Locked Bag 1797, Penrith, NSW 2751 (Australia) E-Mail c.best@uws.edu.au 
I turn now to Phonetica's mission, which has been to provide a platform for publication of high-quality phonetics research and theory from a 'communicative phonetic science' perspective, since its inception in the 1950s [e.g., Fischer-Jørgensen, 1964; Kohler, 2008, 2013]. We will maintain continuity on this mission. In my view, communicative phonetic science research seeks to understand how humans exploit the physical medium of natural languages to communicate with each other. We produce and comprehend utterances using a wide array of forms and styles, for which we make impressive use of the many dimensions of phonetic variation in speech to shape our utterances for a broad range of communicative purposes. In navigating this complex landscape, talkers take advantage of the richness and dynamic variability of which the speech medium is capable, in order to convey multiple dimensions of information that range from the linguistic to the paralinguistic to the social-indexical to the musical and beyond. Deepening our current understanding of how and why humans harness phonetic form to serve so many roles in verbal communication relies on open-mindedness and creative thinking. It requires a willingness to cross and even to blur traditional lines between long-standing dichotomies in the field. It thrives on openness to adapting ideas and methods from relevant disciplines beyond linguistic phonetics alone, and it depends as well on reasoned enthusiasm for novel approaches and innovative combinations of techniques from our increasing and ever-improving scientific toolkit.

These are truly exciting times in the field. Novel approaches and analyses are blossoming and are being applied to both classic issues and new concepts in phonetics, many of the latter arising from investigations of phenomena at junctures between traditionally separate disciplines and subdisciplines. I can provide here just a few examples of the burgeoning research directions that are progressively enriching our understanding of phonetics across diverse communicative contexts: research on caregivers' speech to infants, on infants' perceptual attunement to ambient speech, and on young children's growing abilities to recognize spoken words are helping us to better understand how phonological knowledge coalesces through experience with the phonetic patterns of the native language. Research on speech perception and production in second-language learners and bilinguals is expanding our insights into both the benefits and the limitations of productive and perceptual attunement to native speech. Multimodal studies of speech (visual and tactile as well as audio) are making it clear that phonetic information is not solely acoustic in nature, as are complementary findings on the production and perception of analogous gradient and categorical structure in the manual/facial signals used by sign languages. Neuroimaging and ERP measures of brain activation in response to segmental, prosodic, lexical and syntactic properties of utterances are providing a clearer picture of the processes involved in perception and production of spoken language. Moreover, in addition to standard experimental designs and statistical approaches, computational modelling of data, e.g., from speech corpora, as well as meta-analysis and other uses of 'the new statistics' [e.g., Cummings, 2014] are helping us to better discern relationships between systematic phonetic variation and stable phonological structure in speech at multiple levels of organization: segments, timing units, words, prosodic elements and more. These research advances are of paramount interest for practical and clinical applications as well. For example, improvements in the performance of the automated information systems (automated speech recognition and synthesis) and telecommunication technologies that we increasingly rely on in everyday life, as well as in the functionality of devices designed to enhance communication by individuals with hearing or speech/ 
language impairments, depend on quality research from a communicative phonetic science approach.

Findings on these and many other communicative phonetic science topics are often published in disparate journals, which is non-optimal for cross-fertilization or for achieving broader interdisciplinary understanding. We encourage submissions to Phonetica from across the wide array of research approaches to phonetics in communicative contexts, in the belief that including that range within a single journal may help foster new discussions and cross-cutting research questions that will advance the field.

Phonetica will continue to welcome submissions of both theoretically grounded empirical reports and integrative theoretical papers on the many issues encompassed by a communicative phonetic science approach. Phonetica welcomes, as well, proposals from the research community for Special Issue topics and guest editors; it will in addition continue to have members of the scientific editorial team identify and develop timely Thematic Topics issues. The possibility of establishing a Discussion Forum as a novel format is also under consideration by Phonetica's scientific team; we will announce our decision in the coming months. Most basically, however, we continue to see the publication of individual contributed articles as Phonetica's primary goal.

I look forward to receiving your submissions, and I welcome your thoughts, questions and suggestions.

Catherine T. Best

\section{References}

Cummings, G.: The new statistics: why and how. Psychol. Sci. 75: 7-29 (2014).

Fischer-Jorgensen, E.: Phonometry. Phonetica 11: 144-154 (1964).

Kohler, K.J.: Editorial: the scientific goal of Phonetica. Phonetica 65: 189-193 (2008).

Kohler, K.J.: Farewell. Phonetica 70: 242-246 (2013).

Pavlík, R.: On some quantitative properties of Czech ř in newsreading. Phonetica 71: 22-49 (2014).

Tyler, M.D.; Best, C.T.; Faber, A.; Levitt, A.G.: Perceptual assimilation and discrimination of non-native vowel contrasts. Phonetica 71: 4-21 (2014). 